\title{
Electrochemical Behaviour of Copper Nitroprusside Generated in situ Onto the Graphite Paste Electrode Surface, and its Application in the Determination of N-Acethylcysteine
}

\author{
D. Ribeiro do Carmo, ${ }^{*}$ R. Moutinho da Silva, ${ }^{1}$ N. Ramos Stradiotto ${ }^{1}$ \\ Departamento de Física e Química, UNESP-Universidade Estadual Paulista, Av. Brasil, 56- \\ Centro, C. P. 31, 15385-000 Ilha Solteira- SP, Brazil \\ ${ }^{1}$ Instituto de Química de Araraquara, Universidade Estadual Paulista (UNESP) CP 355, \\ 14801-970 Araraquara-SP, Brazil.
}

Received 10 January 2005; accepted in revised form 10 October 2005

\begin{abstract}
Copper nitroprusside (CuNP) was generated on the graphite paste electrode using a new methodology of the preparation. The electrochemical studies were carried out by cyclic voltammetry technique. The cyclic voltammogram of the modified electrode, CuNP showed two redox couples $\left(\mathrm{E}^{0}\right)_{1}=0.22$ and $\left(\mathrm{E}^{0^{\prime}}\right)_{2}=0.88 \mathrm{~V}$ vs. SCE attributed to $\mathrm{Cu}^{(\mathrm{I})} / \mathrm{Cu}^{(\mathrm{II})}$ and $\mathrm{Fe}^{(\mathrm{II})}(\mathrm{CN})_{5} \mathrm{NO} / \mathrm{Fe}^{(\mathrm{IIII})}(\mathrm{CN})_{5} \mathrm{NO}$, respectively. The nature of the cation affect the $\left(\mathrm{E}^{0^{\prime}}\right)_{1}$ and $\left(\mathrm{E}^{0^{\prime}}\right)_{2}$, as current intensity, shifting the $\mathrm{E}^{0^{\prime}}$ for more positive potentials, for two redox processes. The voltammograms obtained with different $\mathrm{KCl}$ concentrations $\left(0.1-3.0 \mathrm{~mol} \mathrm{~L}^{-1}\right)$ exhibit a shift in the $\left(\mathrm{E}^{0^{3}}\right)_{1}$ to more positive potentials; this change was linear with the supporting electrolyte concentrations change. It was verified that the $\left(\mathrm{E}^{0}\right)_{1}$ remained practically constant at $\mathrm{pH}$ between 6 and 3. However, a new process with $\left(\mathrm{E}^{0}\right)_{3}(0.48 \mathrm{~V})$ appears at $\mathrm{pH}<3$ and it was ascribed to formation of intermediary species.

The redox couple at $\left(\mathrm{E}^{0^{\prime}}\right)_{2}=0.88 \mathrm{~V}$ presents an electrocatalytic response for $\mathrm{N}$ acethylcysteine. The modified graphite paste electrode gives a linear response between $5.0 \times 10^{-4}$ to $1.0 \times 10^{-2} \mathrm{~mol} \mathrm{~L}^{-1}$ of $\mathrm{N}$-acethylcysteine with a detection limit of $4.5 \times 10^{-4}$ $( \pm 5 \%) \mathrm{mol} \mathrm{L}^{-1}(\mathrm{n}=3)$ and an amperometric sensitivity of $4.9 \mathrm{~mA} / \mu \mathrm{mol} \mathrm{L} \mathrm{L}^{-1}$. The electrocatalytic oxidation of $\mathrm{N}$-acethylcysteine compounds by the mediator has been used for the determination of $\mathrm{N}$-acethylcysteine in a commercially pharmaceutical available product.
\end{abstract}

Keywords: copper nitroprusside, modified electrode, electrocatalysis, Nacethylcysteine, oxidation.

\footnotetext{
* Corresponding author. E-mail address: docarmo@fqm.feis.unesp.br
} 


\section{Introdução}

O desenvolvimento de métodos para aplicações eletroanalíticas de eléctrodos modificados, continua sendo uma área em grande desenvolvimento, quer na determinação de analitos orgânicos quer de analitos inorgânicos. Os modificadores imobilizados na superfície eletródica tem sido empregados principalmente para duas finalidades: pré-concentração ou eletrocatálise.

$\mathrm{Na}$ área de eletroanálise demonstra-se que eletrodos quimicamente modificados possuem algumas vantagens sobre eléctrodos convencionais, contribuindo para desenvolvimento de inúmeras aplicações eletroanalíticas, nas quais se incluem os sensores eletroquímicos [1-10].

As propriedades dos eléctrodos modificados dependem do processo de preparação e das características do filme formado na superfície do eléctrodo. Uma das principais vantagens dos eléctrodos quimicamente modificados é a sua capacidade em catalisar a oxidação de algumas espécies que exibem elevado potencial em eléctrodos convencionais, aumentando assim sua sensibilidade e selectividade [11-13]. Possuem vantagens distintas sobre os eléctrodos de mercúrio, que são tóxicos e passíveis de deterioração [14], e sobre os eléctrodos de platina e ouro os quais formam óxidos em suas superfícies causando complicações quando aplicados analiticamente [15].

A dificuldade encontrada na preparação de filmes de nitroprussiato de metais de transição, tais como, $\mathrm{Cu}(\mathrm{II}), \mathrm{Ni}(\mathrm{II})$ e $\mathrm{Co}(\mathrm{II})$ é compatível com o pequeno número de trabalhos existente na literatura. Muitos problemas surgem na sua utilização, tais como, pequena estabilidade eletroquímica e principalmente falta de reprodutibilidade na sua formação, sendo este último aspecto motivo de grandes controvérsias [16].

Recentemente descreveu-se a síntese e a caracterização do nitroprussiato de ferro (Fe(II)NP) [17] e sua aplicação na determinação eletrocatalítica de ácido ascórbico [18] e compostos sulfídricos em fármacos [19]. O eléctrodo modificado contendo Fe(II)NP, obtido por precipitação directa, apresentou uma elevada estabilidade química e eletroquímica. Entretanto, o mesmo procedimento de preparação efectuado para o $\mathrm{CuNP}$, não resultou em um eléctrodo com as mesmas características descritas para o Fe(II)NP.

Embora a estabilidade fosse inicialmente boa, ao contrário do comportamento eletroquímico do $\mathrm{Fe}(\mathrm{II}) \mathrm{NP}$, com o decorrer do tempo verifica-se o aparecimento de alguns picos e o desaparecimento de outros. Estas complicações geradas, ocorrem provavelmente pelo fato do cobre ser um íon metálico bastante lábil e conseqüentemente susceptível a reações paralelas. Este comportamento tem sido considerado como um fator negativo na utilização deste eléctrodo em determinações analíticas.

Visando estabelecer uma nova alternativa metodológica, neste trabalho foi investigado a preparação de um eléctrodo de pasta de grafite contendo nitroprussiato de sódio o qual reage com os íons de cobre em solução, formandose assim o complexo insolúvel na superfície do eléctrodo. $O$ interesse em preparar o complexo in situ na superfície do eléctrodo consiste em melhorar a performance eletroquímica, não adquirida quando o mesmo é sintetizado, 
conforme supra citado. Adicionalmente foi explorado neste trabalho a aplicação directa das capacidades eletrocatalíticas do nitroprussiato de cobre (CuNP) na determinação da $\mathrm{N}$-acetilcisteína em um fármaco.

A $\mathrm{N}$-acetilcisteína é um derivado do aminoácido cisteína e portanto tem um papel importante no sistema vivo. É um fármaco mucolítico empregado no tratamento de doenças respiratórias, e seu grupo sulfídrico $(\mathrm{S}-\mathrm{H})$ reage com os grupos disulfeto presentes nas mucoproteinas para fragmentá-las em unidades menores, diminuindo assim a viscosidade do muco [20].

\section{Parte experimental}

\section{$\underline{\text { Reagentes }}$}

Todos os reagentes utilizados foram de pureza analítica (p.a Merck), sendo a água deionizada utilizando o sistema Milli-Q Gradiente da Millipore. As soluções de $\mathrm{N}$-acetilcisteína foram preparadas imediatamente antes do uso.

Medidas eletroquímicas

Para as medidas de voltametria cíclica empregou-se um potenciostato /galvanostato da Auto Lab (PGSTAT 30). O sistema eletroquímico empregado foi constituído de três eléctrodos: o eléctrodo auxiliar de platina, o eléctrodo de referência de calomelano saturado (ECS) e o eléctrodo de trabalho de pasta de grafite. O eléctrodo de trabalho consiste de um tubo de vidro de $15 \mathrm{~cm}$ de comprimento com diâmetro interno de $0,30 \mathrm{~cm}$ e diâmetro externo de $0,5 \mathrm{~cm}$ sendo a cavidade interna conectada através de um fio de cobre para estabelecimento do contato elétrico.

A técnica de voltametria cíclica foi empregada para o estudo do comportamento eletroquímico. A corrente catalítica foi estabelecida pela diferença entre a corrente medida na presença de $\mathrm{N}$-acetilcisteína e na sua ausência. As soluções foram desaeradas com nitrogênio durante 10 minutos antes da realização das medidas. Os experimentos eletroquímicos foram efetuados a temperatura ambiente.

Preparação do eletrodo de pasta de grafite modificado com CuNP

A pasta de grafite modificada com nitroprussiato de sódio (NP) foi preparada contendo $5 \% \mathrm{~m} / \mathrm{m}$ (5 mg de NP com $95 \mathrm{mg}$ de grafite em pó (Aldrich)) e $50 \mu \mathrm{L}$ de óleo mineral).

Determinação voltamétrica de N-acetilcisteína

$\mathrm{Na}$ determinação de $\mathrm{N}$ - acetilcisteína, preparou-se uma solução padrão $0.1 \mathrm{~mol} \mathrm{~L}{ }^{-}$

${ }^{1}$ de $\mathrm{N}$ - acetilcisteína (solução estoque). O fármaco em forma de tabletes (5.5) foi dissolvido em água deionizada e a solução diluída quantitativamente a $50 \mathrm{~mL}$.

As amostras foram determinadas empregando o método de adição padrão. $\mathrm{O}$ procedimento consistiu em adicionar a uma cela eletroquímica contendo $25 \mathrm{~mL}$ de $\mathrm{KCl} 1.0 \mathrm{~mol} \mathrm{~L}^{-1}$ ( $\mathrm{pH} 3$ ), uma alíquota $(2 \mathrm{~mL}$ ) da amostra (Euroforma), em seguida três alíquotas $(0.5 ; 1,2 \mathrm{~mL})$ de solução padrão de $\mathrm{N}$-acetilcisteína $(0.1$ mol L $\left.{ }^{-1}\right)$. Os experimentos foram realizados em triplicata. 


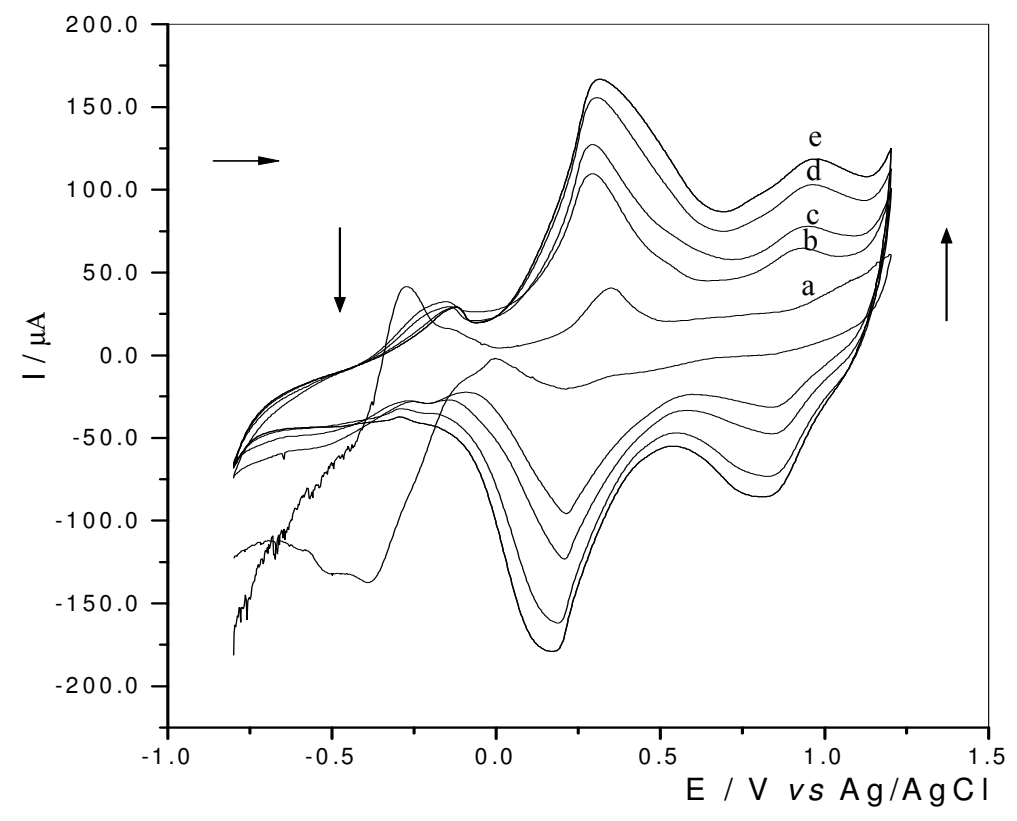

Figura 1. Formação do CuNP na superfície do eletrodo de pasta de grafite em solução de $1.0 \times 10^{-3} \mathrm{~mol} \mathrm{~L}^{-1}$ de $\mathrm{CuCl}_{2}(\mathrm{pH} 3)$ : a ) 1 ; b) 15 ; c) 30 ; d) 75 ; e) 105 ciclos $(5 \%$ $\mathrm{m} / \mathrm{m} ; \mathrm{v}=100 \mathrm{mV} \mathrm{s}^{-1}$ ).

\section{Resultados e discussão}

Comportamento eletroquímico do eléctrodo de pasta de grafite modificado com $\underline{\text { CuNP }}$

O processo de preparação do eléctrodo de pasta de grafite modificado, consistiu primeiramente em fixar a porcentagem do nitroprussiato de sódio (5\%) e variar as concentrações de soluções de $\mathrm{CuCl}_{2}$ em pH 3.0. A Fig. 1 ilustra o processo de formação do CuNP na superfície do eléctrodo de pasta de grafite em solução de concentrações $1.0 \times 10^{-3} \mathrm{~mol} \mathrm{~L}^{-1}$ de $\mathrm{CuCl}_{2}$. Nesta concentração de cobre $\mathrm{o}$ voltamograma do primeiro ciclo exibiu dois pares redox (I) e (II). Estes pares redox forma atribuídos as espécies eletroativas livres antes de ocorrer a complexação.

A partir do $15^{\circ}$ ciclo observa-se a formação de um terceiro par redox (pico III) e o desaparecimento do pico (I). Os pares redox (II) e (III) foram atribuídos ao complexo binuclear formado.

Concomitante a formação do complexo insolúvel CuNP, nas ciclagens sucessivas, verificou-se um pequeno deslocamento do potencial do par redox do pico (I) e do pico (II), tendo o primeiro se deslocado para regiões anódicas e o segundo para regiões catódicas.

As intensidades de corrente observadas para o eléctrodo de CuNP em presença das diferentes concentrações de $\mathrm{CuCl}_{2}$ são praticamente similares, demonstrando 
que a maioria dos sítios disponíveis para complexação foram alcançados mesmo em soluções mais diluídas.

Após a formação do filme de CuNP, o eléctrodo é retirado da célula e lavado com $\mathrm{KCl} 1.0 \mathrm{M}$ ( $\mathrm{pH} 3$ ). O CuNP em pasta de grafite, apresenta dois pares redox distintos com potenciais médios $\left(\mathrm{E}^{0}\right)_{1}$ e $\left(\mathrm{E}^{0^{\prime}}\right)_{2}$ em 0.22 e $0.88 \mathrm{~V}$ vs. Ag/AgCl. O primeiro é atribuído ao processo de oxidação do par $\mathrm{Cu}(\mathrm{I}) / \mathrm{Cu}(\mathrm{II})$ e o segundo ao processo de oxidação $\mathrm{Fe}(\mathrm{II})(\mathrm{CN})_{5} \mathrm{NO} / \mathrm{Fe}(\mathrm{III})(\mathrm{CN})_{5} \mathrm{NO}$ do complexo binuclear formado. A Fig. 2 representa os voltamogramas do eléctrodo modificado mostrando a alta estabilidade eletroquímica alcançada, uma vez que as correntes anódicas e catódicas permanecem praticamente inalteradas após 100 ciclos. Esta característica aliada a boa performance voltamétrica é característica imprescindível para o uso destes eléctrodos em eletroanálise.

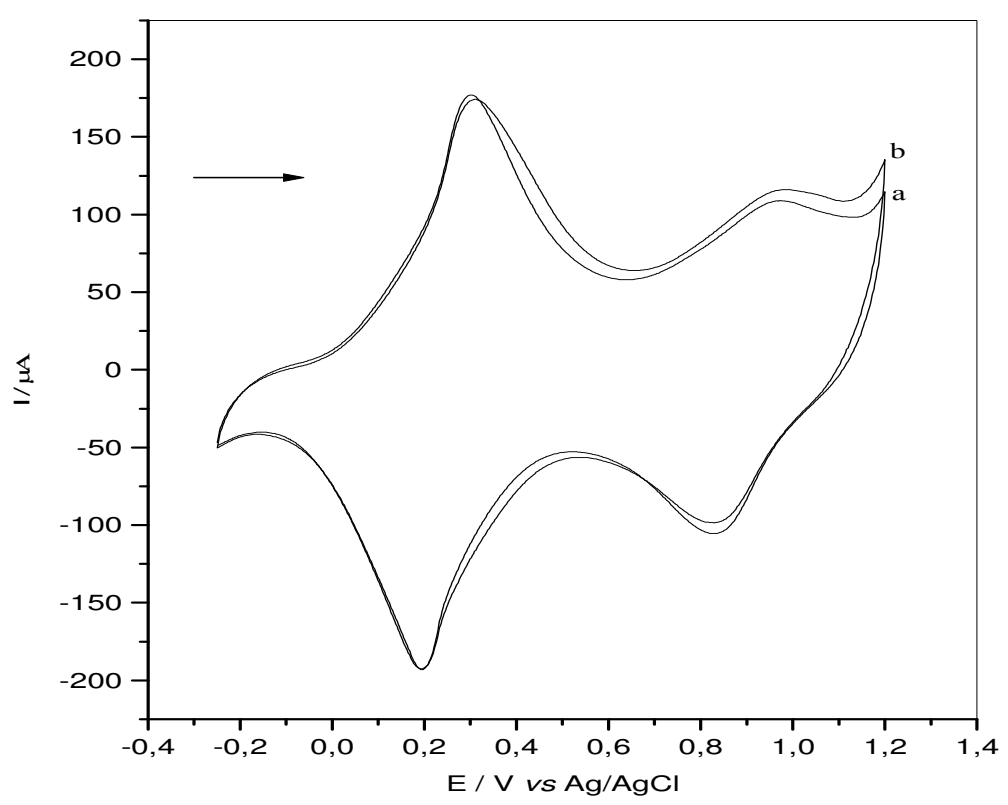

Figura 2. Voltamogramas cíclicos do eléctrodo de pasta de grafite modificado com CuNP obtido com nova metodologia em solução de $\mathrm{KCl} 1.0 \mathrm{~mol} \mathrm{~L}^{-1}$ após (a $=100$ ciclos, $\mathrm{b}=1$ ciclo $) ;\left(\mathrm{v}=100 \mathrm{mV} \mathrm{s}^{-1}\right)$.

Os voltamogramas cíclicos de pasta de grafite modificada com CuNP obtidos em diferentes velocidades de varredura estão ilustrados pela Fig. 3. Para ambos processos encontrou-se uma dependência linear entre a corrente de pico com a velocidade de varredura, o que caracteriza um processo adsortivo [21].

Efeito de cátions e ânions

Nos estudos realizados com diferentes cátions observou-se que, conforme ilustra a Fig. 4, não só a intensidade de corrente como também o potencial médio dos pares redox dos dois processos $\left(\mathrm{E}^{0}\right)_{1}$ e $\left(\mathrm{E}^{0}\right)_{2}$ são influenciadas pela natureza dos 
cátions, sendo que estes potenciais são deslocados para regiões mais positivas na seqüência: $\mathrm{K}^{+}>\mathrm{Li}^{+} \sim \mathrm{Na}^{+}$.

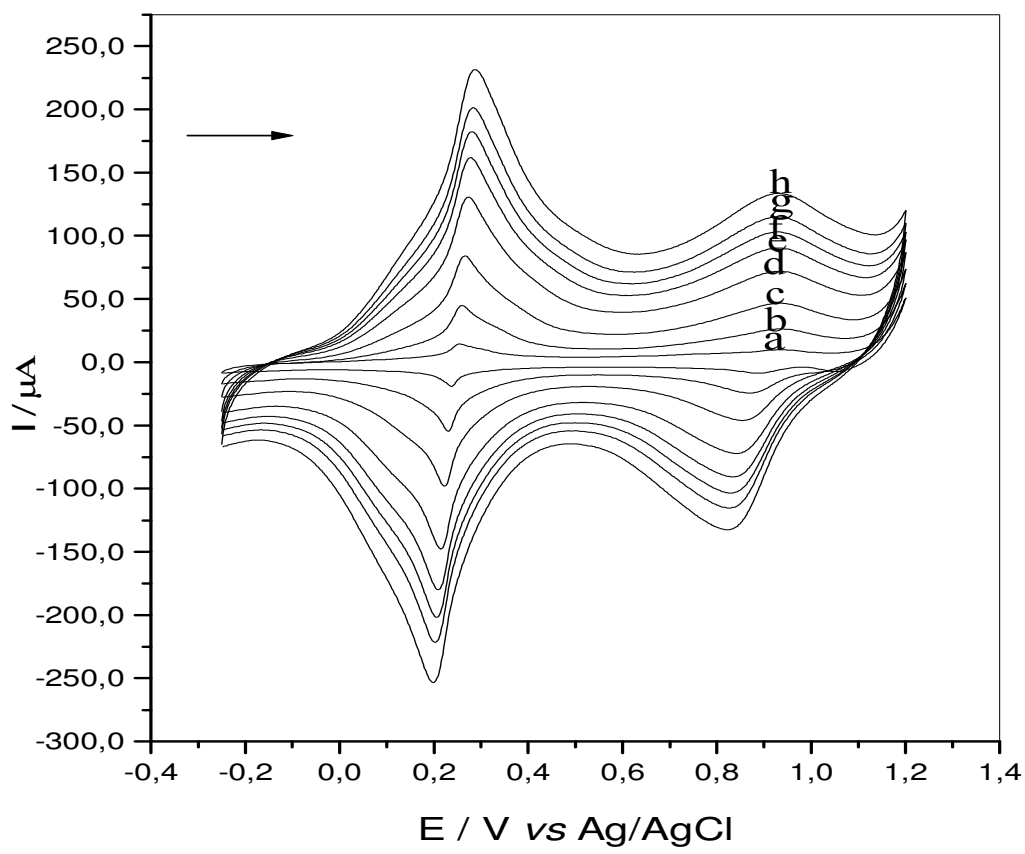

Figura 3. Voltamograma cíclico da pasta de grafite modificada com $\mathrm{CuNP}$ em $\mathrm{KCl} 1.0$ mol L- em diferentes velocidades de varredura (a) 10; b) 30; c) 60); d)100; e) 130; f) 150; g) 170; h) $200 \mathrm{mV} \mathrm{s}^{-1}$ ).

Os voltamogramas cíclicos do eléctrodo de pasta de grafite modificado com CuNP registrados em presença de diferentes ânions, indicaram que os dois processos redox não são fortemente influenciados pela natureza do ânion $\left(\mathrm{Cl}^{-}\right.$, $\mathrm{NO}_{3}^{-}, \mathrm{SO}_{4}{ }^{-}$).

O potencial médio do segundo par redox não é praticamente alterado, quando se emprega $\mathrm{LiCl}$ e $\mathrm{KCl}$ como eletrólitos suporte; entretanto o potencial médio do primeiro par redox é afetado, conforme mostram os valores listados na Tabela 1.

Tabela 1. Relação do diâmetro médio do íon hidratado com os potenciais médios de CuNP.

\begin{tabular}{|c|c|c|c|}
\hline Eletrólito & Diâmetro do ion hidratado $(\mathrm{nm})^{*}$ & $\left(\mathrm{E}^{0}\right)_{1}(\mathrm{~V})$ & $\left(\mathrm{E}^{0}\right)_{2}(\mathrm{~V})$ \\
\hline $\mathrm{KCl}$ & 0.24 & 0.22 & 0.88 \\
\hline $\mathrm{NaCl}$ & 0.36 & 0.12 & 0.94 \\
\hline $\mathrm{LiCl}$ & 0.42 & 0.13 & 0.86 \\
\hline
\end{tabular}

$*$ Ref [22]. Eletrólito $0.5 \mathrm{mo} \mathrm{L}^{-1} ; \mathrm{T}=25^{\circ} \mathrm{C} ; \mathrm{v}=30 \mathrm{mV} \mathrm{s}^{-1}$ 
Assim, não só o tamanho da cavidade, mas também o raio do cátion hidratado presente em solução, são fatores que governam a seletividade deste processo redox.
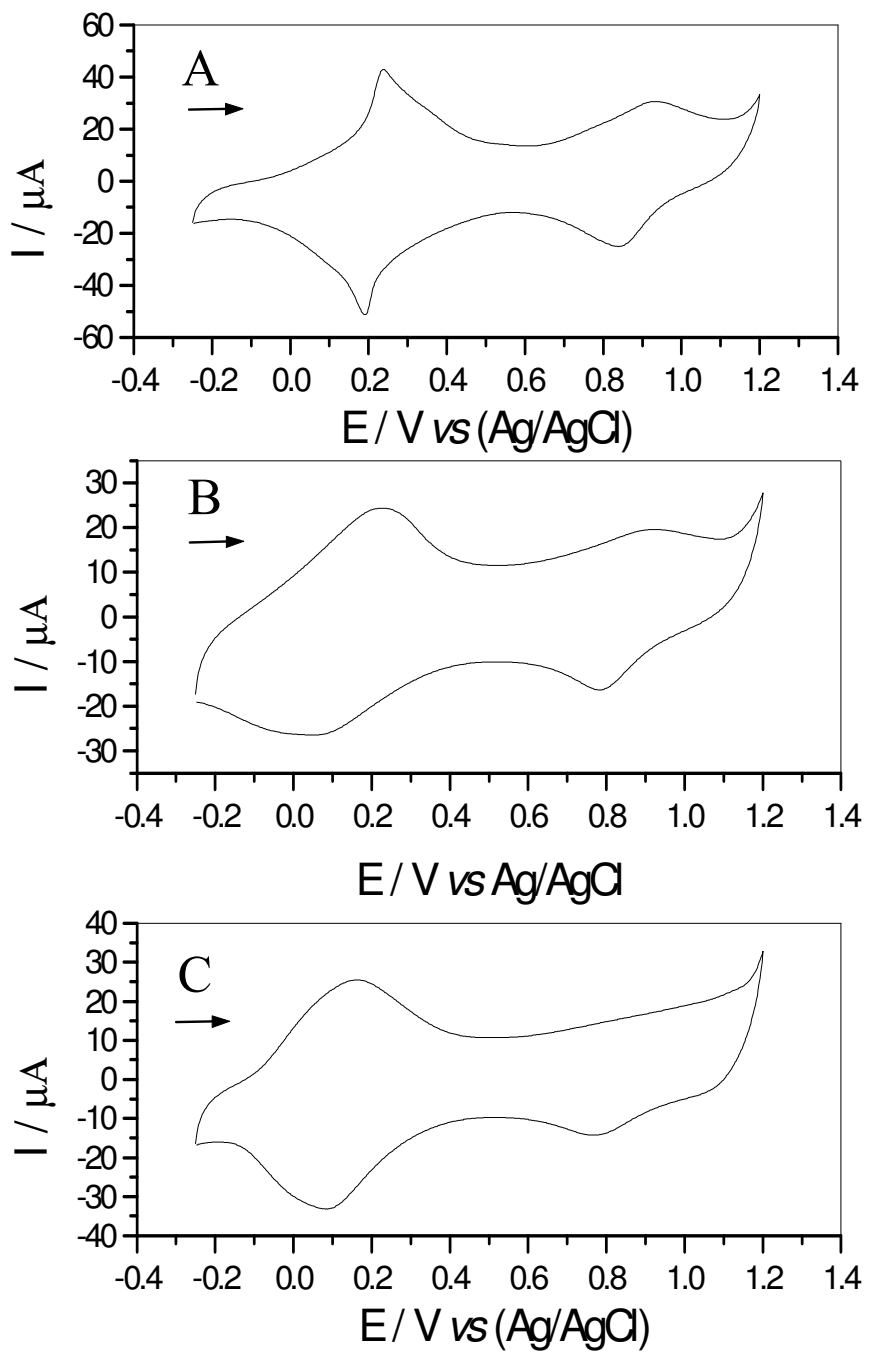

Figura 4. Influência de diferentes cátions na resposta eletroquímica do eléctrodo de pasta de grafite modificada com CuNP: a) $\mathrm{KCl}$; b) $\mathrm{LiCl}$; c) $\mathrm{NaCl}\left(0.5 \mathrm{~mol} \mathrm{~L}^{-1}\right.$; v $=30$ $\left.\mathrm{mV} \mathrm{s}^{-1}\right)$.

Compostos como azul da Prússia e análogos exibem estruturas que apresentam canais que permitem a inserção de pequenas moléculas ou íons. Assim como o ion $\mathrm{K}^{+}$apresenta um raio hidratado menor, este íon poderia sair e entrar na cavidade com maior facilidade que o íon $\mathrm{Li}^{+}$, isto porque o raio hidratado do ion $\mathrm{Li}^{+}$é maior que a cavidade zeolítica, estimada em $0.32 \mathrm{~nm}$ [22], dificultando-se assim a difusão para dentro da cavidade zeolítica, não resultando em uma boa 
resposta eletroquímica. O tamanho das cavidades zeolíticas [23,24] é dependente dos diversos processos de síntese e natureza destes compostos.

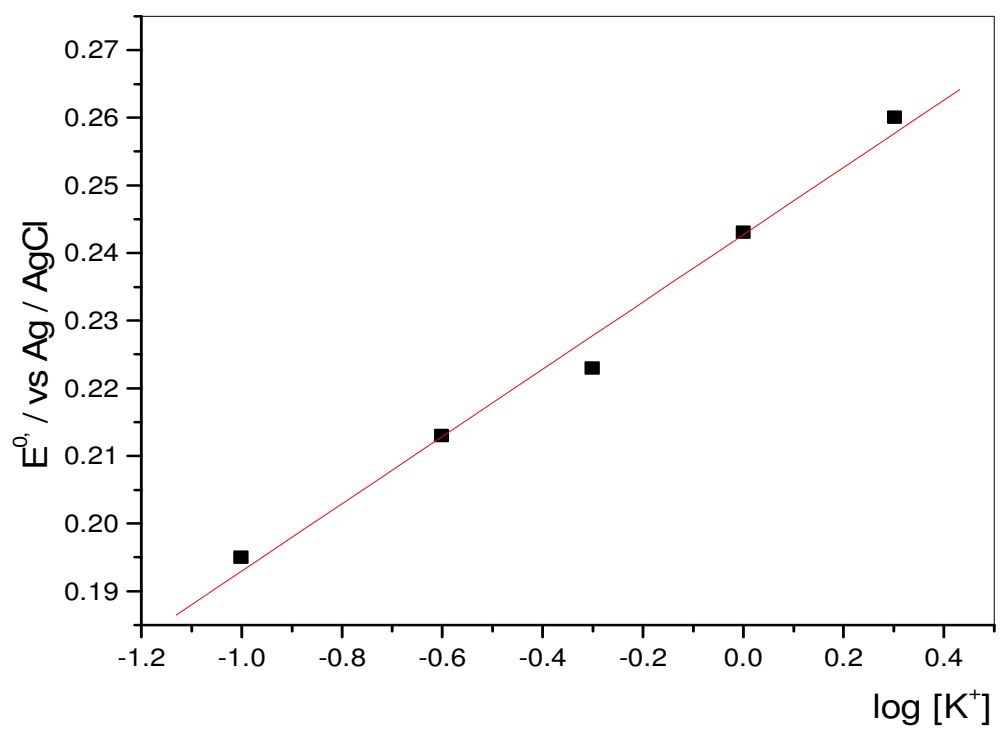

Figura 5. Potencial $\left(\mathrm{E}^{0^{\prime}}\right)_{1}$ da pasta de grafite modificada com CuNP em função da concentração $\left(\mathrm{mol} \mathrm{L}^{-1}\right)$ de íons de $\mathrm{K}^{+} ;\left(\mathrm{v}=30 \mathrm{mV} \mathrm{s}^{-1}\right)$.

Influência da concentração do eletrólito suporte

Estudou-se o comportamento voltamétrico do eléctrodo de pasta de grafite modificado com CuNP em diferentes concentrações de eletrólito suporte. Observa-se que os voltamogramas obtidos em diferentes concentrações do mesmo eletrólito suporte mostram um ligeiro deslocamento do potencial anódico dos dois pares $\left(\mathrm{E}^{0^{\prime}}\right)_{1}$ e $\left(\mathrm{E}^{0^{\prime}}\right)_{2}$ para regiões mais anódicas na medida em que se aumenta a concentração do eletrólito. A Fig. 5 mostra que, os valores de $\mathrm{E}^{0}$ deslocam-se linearmente para potenciais mais positivos quando se varia a concentração de potássio de 0.1 a $3.0 \mathrm{M}$. Para o eletrodo de pasta de grafite modificado com CuNP a inclinação desta reta é de $50 \mathrm{mV}$ por década de concentração de íons potássio indicando, um processo quase nernstiano .

Analogamente ao eléctrodo modificado com $\mathrm{Fe}(\mathrm{II}) \mathrm{NP}$ a resposta quase nernstiana verificada para concentração de potássio, indica a participação deste ion no processo redox. A dependência do potencial $\left(\mathrm{E}^{0^{\prime}}\right)$ com a concentração do eletrólito, pode ser explicada pelo equilíbrio existente entre o cátion e o CuNP, o qual foi descrito em publicação anterior [17]. 


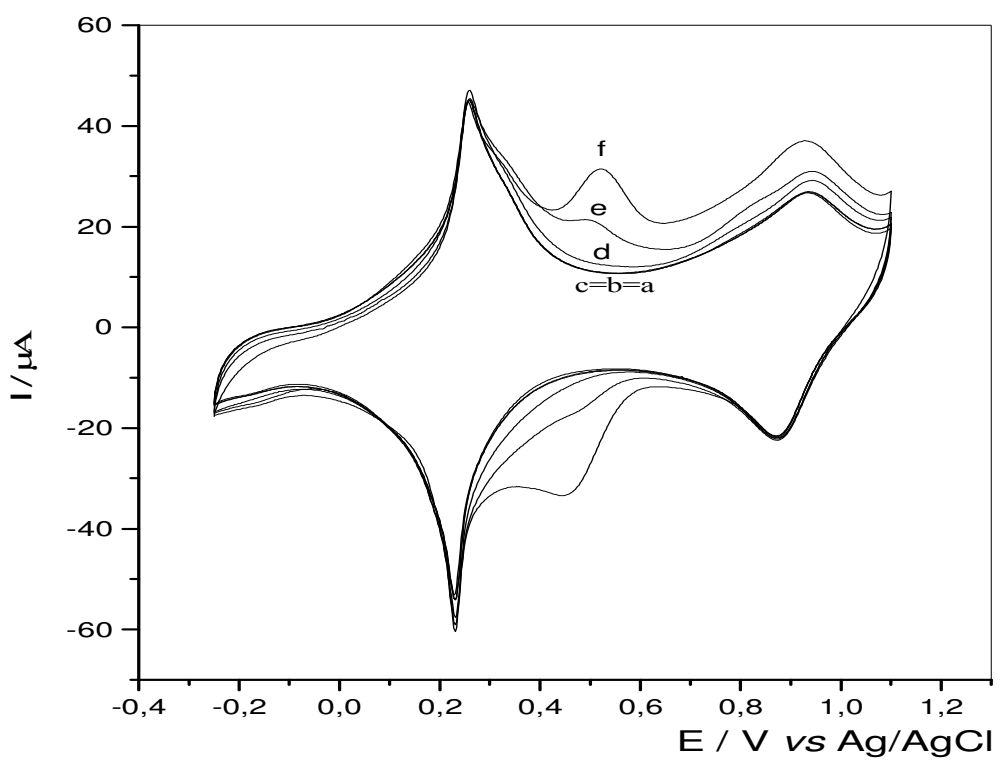

Figura 6. Voltamograma cíclico de pasta de grafite modificada com CuNP em

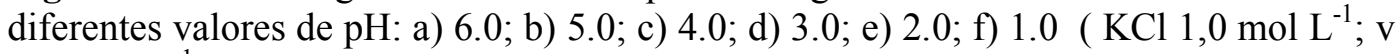
$=30 \mathrm{mV} \mathrm{s}^{-1}$ ).

\section{Influência da concentração hidrogeniônica}

Com a variação da concentração hidrogeniônica (pH 6.0 a 3.0), os dois processos redox $\left(\mathrm{E}^{0^{3}}\right)_{1}$ e $\left(\mathrm{E}^{0^{3}}\right)_{2}$ permanecem quase que inalterados; no entanto, a partir de valores de $\mathrm{pH}$ menores que 3.0 surge um novo processo com $\left(\mathrm{E}^{0^{\prime}}\right)_{3}=0.48 \mathrm{~V}$, conforme ilustra a Fig. 6. Por analogia com azul da Prússia [25] a existência deste novo pico é possivelmente devido a presença de alta concentração de $\mathrm{H}^{+}$, a qual governa a eletroatividade de uma ou mais formas de espécies intermediárias. Este pico também foi detectado no eléctrodo de pasta de grafite modificado com Fe(II)NP [17] em concentração hidrogeniônica alta $(\mathrm{pH}<3)$.

\section{Comportamento eletroquímico}

O comportamento eletroquímico do eléctrodo de pasta de grafite modificado com CuNP para a eletro-oxidação da $\mathrm{N}$-acetilcisteína em $1.0 \mathrm{~mol} \mathrm{~L}^{-1} \mathrm{KCl}$ é ilustrado na Fig. 7. $\mathrm{O}$ eletrodo de pasta de carbono não apresenta qualquer par redox na escala de potencial entre -0.3 e $1.2 \mathrm{~V}$ na ausência e presença de $\mathrm{N}$-acetilcisteína ( curva a, b respectivamente). Após a adição de $\mathrm{N}$-acetilcisteína observou-se um aumento na intensidade de corrente do pico anódico (curva d).

A intensidade da corrente do pico anódico aumenta proporcionalmente em relação a concentração do analito. Este aumento da intensidade da corrente anódica ocorre devido a oxidação eletrocatalítica da $\mathrm{N}$-acetilcisteína pelo mediador CuNP. O Fe(III) produzido durante a varredura anódica oxida quimicamente a $\mathrm{N}$-acetilcisteína, enquanto que este é reduzido a $\mathrm{Fe}(\mathrm{II})$, que por sua vez é eletroquimicamente oxidado a Fe(III). 


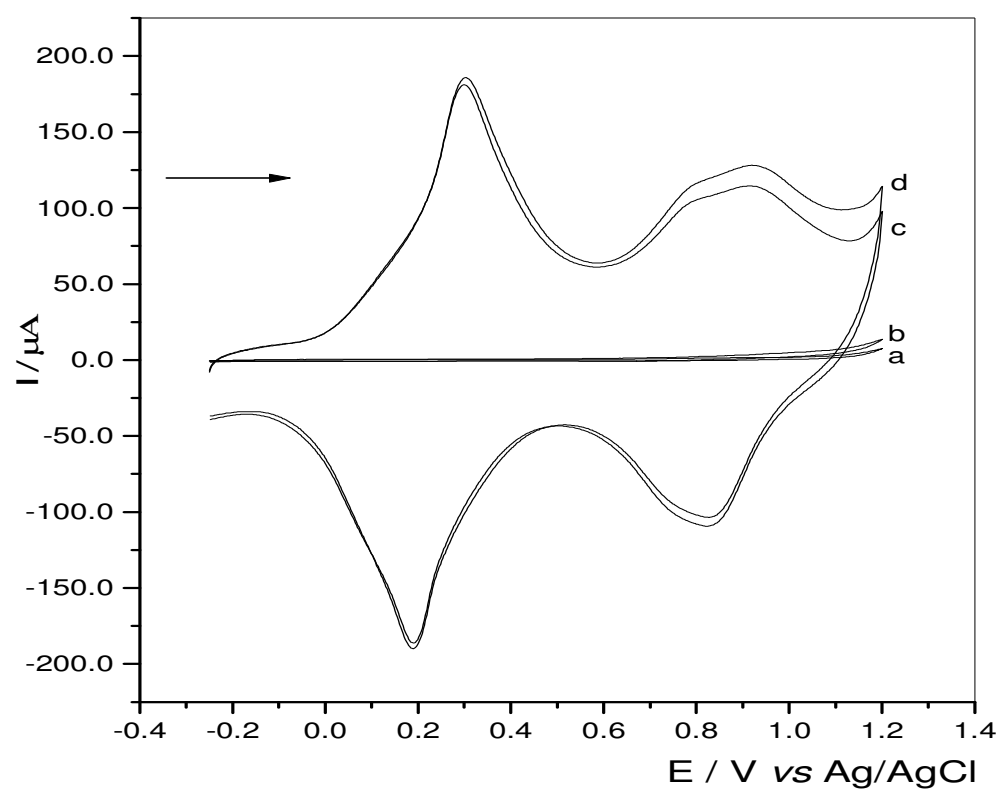

Figure 7. Voltamograma cíclico de: a) pasta de grafite; b) pasta de grafite e $1.0 \times 10^{-3}$ mol L ${ }^{-1} \mathrm{~N}$-acetilcisteína ; c) pasta de grafite modificada com CuNP ; d) pasta de grafite modificada com CuNP e $1.0 \times 10^{-3} \mathrm{~mol} \mathrm{~L}^{-1} \mathrm{~N}$-acetilcisteína $\left(1.0 \mathrm{~mol} \mathrm{~L} \mathrm{KCl}^{-1} \mathrm{v}=100\right.$ $\left.\mathrm{mVs}^{-1}\right)$.

A equação da oxidação eletroquímica da L-cisteina, descrita na literatura [26], pode ser representada da seguinte forma:

$$
2 \mathrm{~L}-\text { cisteína }(\mathrm{CySH}) \rightarrow \text { cistina }(\mathrm{CySSCy})+2 \mathrm{e}+2 \mathrm{H}^{+}
$$

Logo, tendo em vista esta reação da L-cisteína, a oxidação da $\mathrm{N}$-acetilcisteína na superficie eletródica do eléctrodo de pasta de grafite modificado com CuNP pode ser descrita pelas seguintes reações :

$$
\begin{gathered}
\left.\left.\mathrm{Cu}^{\mathrm{I}}\left[\mathrm{Fe}^{\mathrm{II}} \mathrm{CN}\right)_{5} \mathrm{NO}\right] \rightleftarrows \mathrm{Cu}^{\mathrm{II}}\left[\mathrm{Fe}^{\mathrm{II}} \mathrm{CN}\right)_{5} \mathrm{NO}\right](0.22 \mathrm{~V})+e \\
\left.\left.\mathrm{Cu}^{\mathrm{II}}\left[\mathrm{Fe}^{\mathrm{II}} \mathrm{CN}\right)_{5} \mathrm{NO}\right] \rightleftarrows \mathrm{Cu}^{\mathrm{II}}\left[\mathrm{Fe}^{\mathrm{III}} \mathrm{CN}\right)_{5} \mathrm{NO}\right](0.88 \mathrm{~V})+\mathrm{e} \\
\left.\mathrm{Cu}^{\mathrm{Il}}\left[\mathrm{Fe}^{\mathrm{III}} \mathrm{CN}\right)_{5} \mathrm{NO}\right]+2 \mathrm{X} \\
\left.\rightleftarrows \mathrm{Cu}^{\mathrm{II}}\left[\mathrm{Fe}^{\mathrm{II}} \mathrm{CN}\right)_{5} \mathrm{NO}\right]+\mathrm{Y}+2 \mathrm{H}^{+}
\end{gathered}
$$

onde $\mathrm{X}=\mathrm{N}$-acetilcisteína e $\mathrm{Y}=$ é $\mathrm{N}$-acetilcistina

Desta forma $\mathrm{N}$-acetilcisteína é oxidada na superficie eletródica, sendo que este processo ocorre no potencial de $0.88 \mathrm{~V}$. O processo de oxidação não ocorre neste potencial quando se emprega eléctrodo de carbono vítreo ou mesmo o eléctrodo de pasta de grafite não modificado. $\mathrm{O}$ potencial do pico não é afetado pela concentração da $\mathrm{N}$-acetilcisteína e a corrente catalítica é também linear com a velocidade de varredura.

$\mathrm{O}$ comportamento eletroquímico da oxidação da $\mathrm{N}$-acetilcisteína em eléctrodo modificado com o CuNP difere do eléctrodo modificado com o filme do azul da Prússia, o qual exibe um aumento de corrente nos dois potenciais de oxidação 
$(0.79$ e $0.95 \mathrm{~V})$ quando na presença de $\mathrm{N}$-acetilcisteína $\left(1.0 \mathrm{~mol} \mathrm{~L}{ }^{-1} \mathrm{KCl}, \mathrm{pH} 3.5\right.$ vs. $\mathrm{Ag} / \mathrm{AgCl}$ ) [27]. No entanto apresentou comportamento similar ao eléctrodo de pasta de carbono modificado com nitroprussiato de ferro (Fe(II)NP), o qual também mostrou somente acréscimo de corrente catalítica no pico de oxidação correspondente ao potencial em $0.88 \mathrm{~V}$ (vs. SCE).

Por outro lado, o filme de azul da Prússia [27] é degradado pela reação de hidrólise em soluções com valores de $\mathrm{pH}$ muito acima de cinco, enquanto que no filme de CuNP a hidrólise ocorre em valores de $\mathrm{pH}$ maiores que sete.

Semelhantemente ao Fe(II)NP, o eléctrodo com CuNP mostra que em valores de pH menores que 2.5 a elevada concentração protônica promove o aparecimento de um pico adicional intermediário [17]. No entanto a corrente catalítica da Nacetilcisteína neste eléctrodo (FeNP) decresce com o tempo depois do primeiro ciclo, provavelmente devido a depleção [26] próxima a superfície eletródica ou então a resposta do eléctrodo é bloqueada pelo produto resultante da reação [28], o que não acontece com o eléctrodo modificado com CuNP.

A Fig. 8 ilustra a curva analítica da concentração de $\mathrm{N}$-acetilcisteína em função da corrente anódica. O eletrodo modificado apresenta uma região linear no intervalo de concentração de $5.0 \times 10^{-4}$ a $1.0 \times 10^{-2} \mathrm{~mol} \mathrm{~L}^{-1}$ tendo uma equação correspondente a $\mathrm{Y}(\mu \mathrm{A})=1.688+4918$ [N-acetilcisteína] com um coeficiente de correlação de 0.998 . O método apresenta um limite de detecção de $4.5 \times 10^{-4} \mathrm{M}$ com uma sensibilidade amperométrica de $4.9 \mathrm{~mA} / \mu \mathrm{mol} \mathrm{L}{ }^{-1}$.

A repetibilidade apresentada mostrou um RSD de 5\% $(n=3)$ no intervalo de concentração estudado. Outra característica do eléctrodo de pasta de carbono modificado com CuNP, é que o mesmo pode ser empregado seguidamente, lavando-se o eléctrodo unicamente com água deionizada, não sendo necessário remover a superfície do eléctrodo.

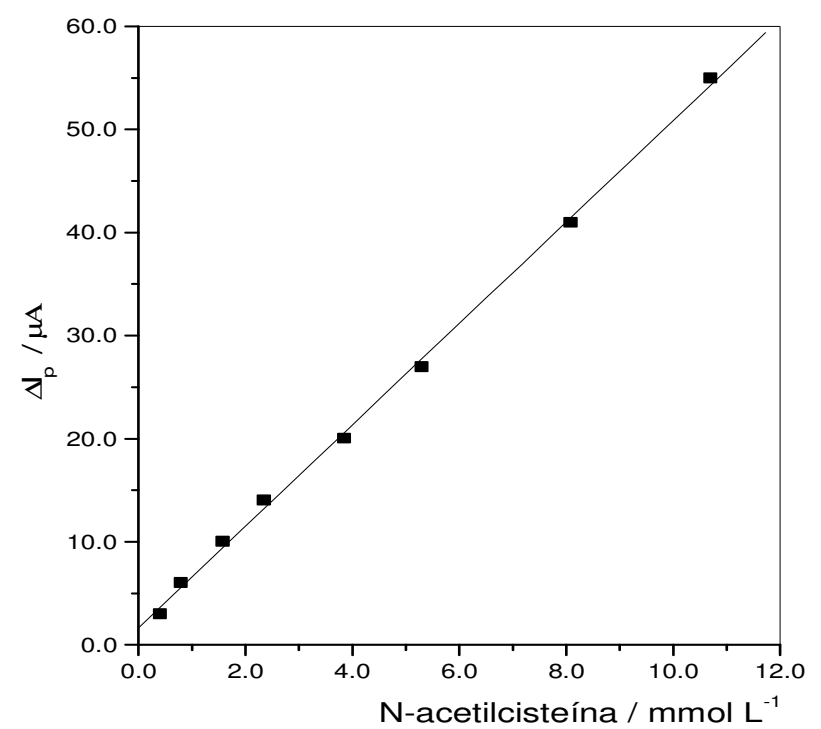

Figura 8. Curva analítica para determinação de $\mathrm{N}$-acetilcisteína empregando o

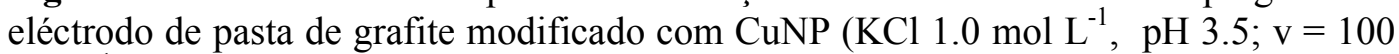
$\mathrm{mV} \mathrm{s}^{-1}$ ). 


\section{Aplicação}

$\mathrm{O}$ eléctrodo de pasta de carbono modificado com CuNP, foi aplicado para a determinação do fármaco Eurofarma utilizando o método de adição padrão. A Fig. 9 ilustra os voltamogramas utilizando este eléctrodo, após a adição do fármaco e de soluções padrão de $\mathrm{N}$-acetilcisteína.

Os valores obtidos pelo presente método para a determinação de $\mathrm{N}$-acetilcisteína no fármaco $(212 \pm 2 \mathrm{mg})(\mathrm{n}=3)$ estão em concordância com a quantidade declarada nas tabletes $(219 \mathrm{mg})$.

\section{Conclusão}

O nitroprussiato de cobre foi gerado in situ, após sucessivas ciclagens, na superfície do eléctrodo de pasta de grafite contendo nitroprussiato de sódio. $\mathrm{O}$ comportamento eletroquímico de $\mathrm{CuNP}$ em pasta de grafite apresentou dois pares redox distintos com potenciais médios $\left(\mathrm{E}^{0^{\prime}}\right)_{1}$ e $\left(\mathrm{E}^{0^{\prime}}\right)_{2}$ nos valores de 0.22 e 0.88 $\mathrm{V}$ vs. Ag/AgCl, respectivamente. Ambos pares redox apresentaram alterações não só com respeito a natureza dos cátions $\left(\mathrm{Li}^{+}, \mathrm{Na}^{+}, \mathrm{K}^{+}\right)$, mas também em relação as variações de concentração. A natureza dos ânions não apresentou influência sobre o comportamento eletroquímico do eléctrodo modificado com CuNP. A dependência linear entre a corrente e a velocidade de varredura indica um processo controlado por adsorção.

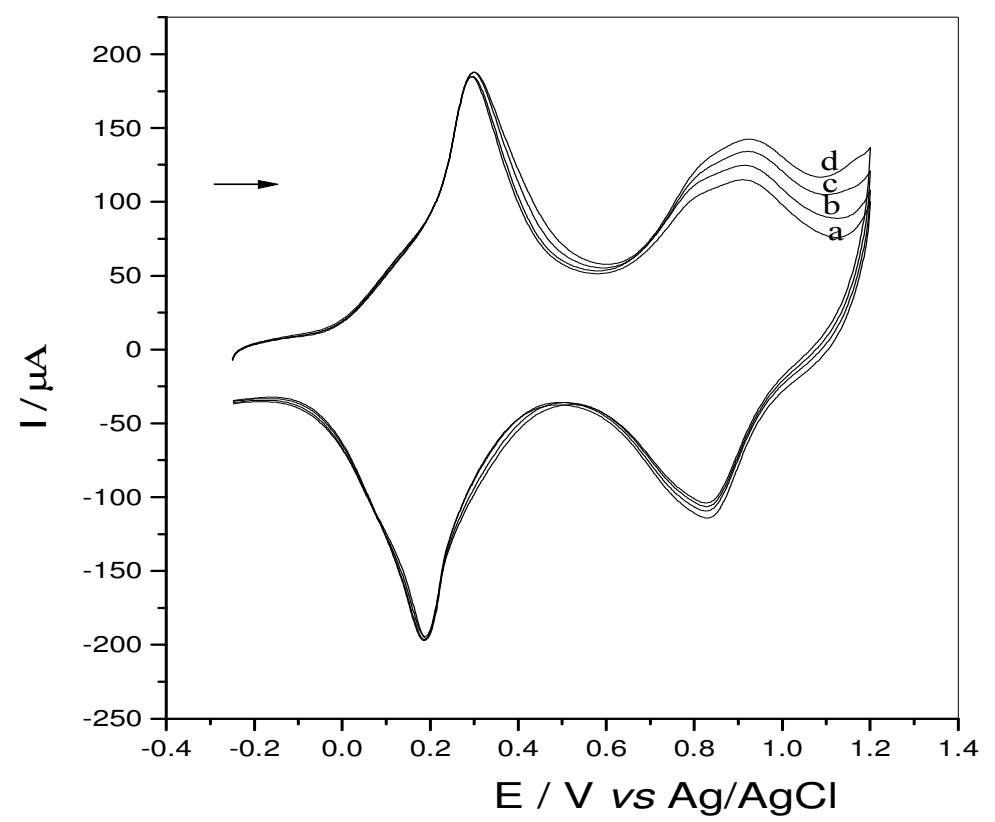

Figura 9. Voltamograma cíclico de: a) eléctrodo de pasta de grafite modificado com CuNP; b) com adição de $2.0 \mathrm{~mL}$ de Eurofarma (amostra); c) com adição de $0.5 \mathrm{~mL}$ de padrão; d) com adição de $1.0 \mathrm{~mL}$ de padrão (concentração da $\mathrm{N}$-acetilcisteína $0.1 \mathrm{~mol}$ $\left.\mathrm{L}^{-1}\right)\left(\mathrm{v}=100 \mathrm{mV} \mathrm{s}^{-1} ; \mathrm{KCl} 1.0 \mathrm{~mol} \mathrm{~L}^{-1} \mathrm{pH} 3.5\right)$. 
$\mathrm{O}$ processo redox $\left(\mathrm{E}^{0^{\prime}}\right)_{2}=0.88 \mathrm{~V}$, mostrado pelo eléctrodo de pasta de grafite modificado com nitroprussiato de cobre CuNP, apresentou atividade eletrocatalítica na oxidação de $\mathrm{N}$-acetilcisteína. $\mathrm{O}$ intervalo linear obtido para a determinação de N-acetilcistína foi de $1.0 \times 10^{-3}$ a $2.6 \times 10^{-2} \mathrm{~mol} \mathrm{~L}^{-1}$ apresentando um limite de detecção de $4.5 \times 10^{-4}$ e uma sensibilidade amperométrica de 4.9 $\mathrm{mA} / \mu \mathrm{mol} \mathrm{L} \mathrm{L}^{-1}$.

A aplicação do eléctrodo de pasta de grafite modificado com CuNP na determinação de $\mathrm{N}$-acetilcisteína em um fármaco (Eurofarma), apresentou valores compatíveis com aqueles indicados no rótulo.

Quando comparada com outros eléctrodos modificados, uma das principais vantagens deste eléctrodo construído empregando a metodologia proposta, é que o mesmo é estável, reprodutível e de fácil confecção.

\section{Referências}

1. Z. Gao, J. Electroanal. Chem. 358 (1993) 161.

2. V.S. Ijeri, P.V. Jaiswal, A.K. Scrivastava, Anal. Chim. Acta 439 (2001) 291.

3. R. Garjonytê, A. Malinauskas, Sensors and Actuators B 46 (1998) 236.

4. A.C. Haubey, B.D. Malhotra, Biosens. Bioelectron. 17 (2000) 441.

5. D.R. Shakaran, S.S. Narayanan, Bull. Electrochem. 4 (1998) 267.

6. $\quad$ S.A. Wring, J.P. Hart, Analyst 117 (1992) 1215.

7. S.M. Chen, J. Electroanal. Chem. 521 (2002) 29.

8. S.M. Golabi, H.R. Zare, M. Hamzehloo, Electroanalysis 14 (2002) 611.

9. N.R. De Toccani, K. Rajeshwar, R.O. Lesna, Electrochimica Acta 45 (2000) 3403.

10. N. Totir, C. Luca, S. Lupu, C. Lete, A.C. Ion Revue Roumaine de Chimie 46 (2001) 555.

11. K. Kalcher, J.M. Kanffmann, J. Wang, J. Scancara, K. Vytras, C. Neuhold, Z. Yang, Electroanalysis 7 (1995) 5.

12. H. Razmi-Nerbin, M.H. Pournaghi-Azar, J. Solid State Electrochem. 6 (2002) 126.

13. T.J. Maflate, T. Nyokong, J. Electroanal. Chem. 408 (1996) 213.

14. R.F. Bergstron, D.R. Kay, J.E. Wagner, J. Chromatogr. 222 (1981) 445.

15. M.E. Jobll, D.G. Williams, D.C. Johnson, Electroanalysis 9 (1997) 1397.

16. V.D. Ivanov, M.M. Kaplum, Russian J. Electrochem. 14 (1998) 559.

17. D.R. do Carmo, R.M. da Silva, N.R. Stradiotto, Ecl. Quim. 27 (2002)197.

18. D.R. do Carmo, R.M. da Silva, N.R. Stradiotto, Port. Electrochim. Acta 22 (2004) 71 .

19. D.R. do Carmo, R.M. da Silva, N.R. Stradiotto, J. Braz. Chem. Soc. 14 (2003) 616.

20. R.P. Tomkiewicz, E.M. App, G.T. de Sanctis, M. Coffiner, P. Mães, B.K. Rubin, M. King, Pulm. Pharm. 8 (1995) 259.

21. A.J. Bard, L.R. Faulkner, Electrochemical Methods, New York: Wiley, 1980.

22. D. Engel, E.W Grabner, Ber. Bunsenges. Phys. Chem. 89 (1985) 982. 
23. M. Jayalakshmi, H. Gomathy, G. P. Rao, Solvent and cationic effects as probes for investigating surface PB films, Bull. Electrochem. 12 (1996) 490.

24. K.D. Karlin, Progress in inorganic chemistry, J. Wiley \& Sons, 45 (1998).

25. N.F. Zakharchuk, B. Meyer, H. Henning, F. Scholz, A. Jaworksi, Z.J. Stojeck, Electroanal. Chem. 398 (1995) 23.

26. T.R. Ralph, M.L. Hitchman, J.P. Millington, F.C. Walsh, J. Electroanal. Chem. 375 (1994) 1.

27. W. Hou, E. Wang, J. Electroanal. Chem. 316 (1991) 155.

28. N. Sãpataru, B.V. Sarada, E. Popa, D.A. Tryk, A. Fujishima, Anal. Chem. $73(2001) 514$. 